Infertility as a result of antineoplastic therapy is becoming a very important issue due to the growing incidence of neoplastic diseases. Routinely applied antineoplastic treatments and the illness itself lead to fertility disorders.

Therapeutic methods used in antineoplastic treatment may cause fertility impairment or sterilization due to permanent damage to reproductive cells. The risk of sterilization depends on the patient's sex, age during therapy, type of neoplasm, radiation dose and treatment area. It is known that chemotherapy and radiotherapy can lead to fertility impairment and the combination of these two gives an additive effect.

The aim of this article is to raise the issue of infertility in these patients. It is of growing importance due to the increase in the number of children and young adults who underwent radiotherapy in the past.

The progress in antineoplastic therapy improves treatment results, but at the same time requires a deeper look at existential needs of the patient. Reproductive function is an integral element of self-esteem and should be taken into account during therapy planning.

Key words: infertility, radiotherapy, chemotherapy.

Contemp Oncol (Pozn) 2016; 20: 199-204 DOI: $10.5114 /$ w0.2016.57814

\section{Fertility impairment in radiotherapy}

\author{
Marta Biedka1, Tamara Kuźba-Kryszak², Tomasz Nowikiewicz³ \\ Agnieszka Żyromska²
}

${ }^{1}$ Chair and Clinic of Oncology and Brachytherapy, Nicolaus Copernicus University in Torun, Ludwik Rydygier Collegium, Medicum in Bydgoszcz, Poland

${ }^{2}$ Radiotherapy Department, Oncology Center, Prof Lukaszczyk Memorial Hospital, Bydgoszcz, Poland

${ }^{3}$ Department of Clinical Breast Cancer and Reconstructive Surgery, Oncology Center, Prof. Lukaszczyk Memorial Hospital, Bydgoszcz, Poland

\section{Introduction}

It is estimated that in 2010 more than 1.5 million people was diagnosed with neoplastic disease, $10 \%$ of whom were younger than 45 years. Retaining fertility and having children is an important aspect of life and antineoplastic therapy for these people [1]. It is estimated that 3/4 of people below the age of 35 who were diagnosed with neoplastic disease and treated during childhood will want to have children. Approximately $81 \%$ of adolescent girls and 93\% of their parents are interested in their children's fertility preservation during antineoplastic therapy [1]. It is estimated that $45 \%$ of physicians do not discuss the issue of fertility preservation and do not suggest appropriate consultations before starting antineoplastic therapy [1, 2].

Therapeutic methods used in antineoplastic treatment cause fertility impairment or sterilization due to permanent damage to reproductive cells [1]. The risk of sterilization depends on patient's age during therapy, type of neoplasm, radiation dose and treatment area. It is known that systemic treatment and radiotherapy can lead to fertility impairment and the combination of these two gives an additive effect [1,3]. Radiotherapy can cause pituitary dysfunction, leading indirectly to impairment of ovarian function, or result directly in inability to conceive and carry a pregnancy to full term [4].

The aim of this article is to raise the issue of infertility in these patients. It is of growing importance due to the increase in the number of children and young adults who underwent radiotherapy in the past. Still improving longterm results of these therapies mean that this issue must be addressed. Additionally, there is only a small number of studies concerning fertility impairment and radiotherapy, and their results are usually extrapolated from experimental studies or case reports.

\section{Effect of radiotherapy on female and male fertility}

Adverse events of radiation therapy associated with fertility impairment affect patients treated with radiation to the area of the head and neck, pelvis and spine. Head and neck radiotherapy can damage the central nervous system, including the hypothalamus and/or pituitary, leading to hyperprolactinemia, gonadotropin deficiency and precocious puberty, causing directly or indirectly impairment of ovarian function. Radiotherapy of pelvis, spine or testicles shows a direct effect on the gonads, resulting in infertility and steroid hormone production disorders [4].

The biological response to radiation varies depending on the organ and tissue type; both the total and the single dose given to the patient are important. An example of this is radiotherapy to the scrotum where a single radiation dose damages germinal epithelium to a lesser extent than the same dose divided into a few fractions. In the setting of the ovaries there is a reverse effect. It is also significant if there was a tissue injury before radiation exposure, e.g. during surgery, as in this situation tissues are dose-depen- 
dent, which means fertility depends on a given radiation dose [4].

\section{Testicle}

The testicle is one of the most radiosensitive tissues, with the lowest dose leading to its dysfunction - a dose of 0.15 Gy leads to a significant decrease in semen volume, and 0.3-0.5 Gy causes temporary oligospermia [5]. Such doses can be received not only during radiation therapy, but also as a result of radiologic imaging. Testicular tissue can be irradiated for prophylaxis radiotherapy of the pelvis and/or abdomen. It is estimated that low doses received per year due to radiation exposure do not have such an unfavorable effect as single high exposure, e.g. due to a nuclear accident, which leads to a long-term decrease in semen volume. As a result of radiotherapy, loss of proliferation in Leydig and Sertoli cells is observed. A dividing spermatogonium is very radiosensitive - a dose below 1 Gy leads to a substantial reduction in the number of spermatogonia and daughter cells. Doses causing death of spermatocytes are higher than in the case of spermatogonia (2-3 Gy); spermatids are not damaged by that dose, but after receiving a 4-6 Gy dose a noticeable decrease in the sperm count can be observed. The spermatocyte and spermatid lifetime is approximately 46 days, and the time needed for a spermatozoon to reach the ejaculate from a seminiferous tubule through the system of efferent ducts is 4 to 12 days. Therefore, during the first 50-60 days the sperm count is reduced to 50\% (at a 1.5-2 Gy dose), and after that period of time it is dramatically reduced, leading even to azoospermia $[4,6]$. The most severe postradiation sperm cell damage occurs between 4 and 6 months after radiotherapy completion [5]. Higher radiation doses lead much faster to extended or permanent oligo- and azoospermia. Return of fertility is a result of proliferation and regeneration of stem cells which have survived. After a single exposure dose, the time to return of normal semen volume and sperm count is 9-18 months for a dose below $1 \mathrm{~Gy}, 30$ months for a dose of 2-3 Gy and 5 or more years for a dose of 4-6 Gy. Daily low fractional doses, which are used nowadays in radiotherapy, lead to protection of cells from late side effects $[1,4,5,7]$.

Speiser et al. assessed 10 patients who received a daily testicular dose of 0.12 Gy and a total dose of 1.4-3.0 Gy. All the patients had azoospermia, but only 2 of them for a longer time than 16 months. After radiation exposure azoospermia can be observed at doses as low as 0.35 Gy. At doses around 2-3 Gy it was germinal epithelium that was damaged, which led to delayed return of spermatogenesis, even to 10 years $[4,9]$.

Tsatsoulis et al. evaluated functions of Leydig and Sertoli cells in 18 men aged 21-49, who underwent orchidectomy and radiotherapy of a total dose of 30 Gy in 20 fractions. Levels of testosterone and gonadotropin were determined in these patients. It was observed that the testosterone level was lower in the patients who underwent radiotherapy than in the control group, but the luteinizing hormone (LH) level was higher. The testosterone/LH ratio was statistically significantly lower, pointing to Leydig cell damage. The authors established that the dose of 24-25 Gy in 12 fractions given directly to the testicle in the treatment of acute lymphoblastic leukemia in boys leads to total ablation and loss of function of Leydig cells immediately after radiotherapy and no return of their function even 5 years after completion of treatment. Most of these patients needed androgen supplementation in order to maintain a normal process of sexual maturation $[4,10]$.

\section{Ovary}

Most of the information concerning fertility preservation after radiation exposure of ovaries was obtained after the atomic bombings of Hiroshima and Nagasaki. Ovaries of newborn girls have approximately 2 million oocytes, and this number decreases with age to around 25 thousand at the age of $37-38$ years $[4,7,11]$. Radiation damages DNA of ovarian cells, leading to its atrophy and a decrease in ovarian reserve, which affects hormone levels and uterus function and leads to earlier menopause [7]. As a result of radiation exposure, oocytes, which are extremely radiosensitive, are either repaired or eliminated by phagocytosis. Differentiation stage of oogonium during the exposure determines whether radiation leads to cell death $[1,7]$. The oogonium in the prenatal period undergoes a great number of mitotic divisions, which affects its radiosensitivity. Sensitivity to radiation injury decreases with subsequent phases of meiotic divisions. There are several factors affecting ovarian fertility impairment: radiation dose, age at time of the exposure and size of the irradiated area [7, 12, 13].

In his mathematical model Wallace et al. suggests that a dose of 2 Gy can lead to destruction of $50 \%$ of immature oocytes $[7,14]$. The effective sterilizing dose (ESD) is a dose which leads to immediate ovarian dysfunction in $97.5 \%$ of the patients after treatment. The ESD decreases with age during treatment: it is 20.3 Gy for newborns; 18.4 Gy for 10-year-old girls; 16.5 Gy for 20-year-old women; 14.3 Gy for 30-year-old women [7, 14, 15]. However, a wide variability of ovarian reserve is observed in women at the same age.

The use of radiation for sterilization in the years 1924 1957 provides information on the effect of low radiotherapy doses on ovaries. The dose received in three sessions was 0.215 Gy to the pituitary and 0.065 Gy to the ovaries. No side effects were observed and 308 out of 794 women gave birth to healthy children [4].

Another study evaluated 1817 women treated with intracavitary brachytherapy due to bleeding from the reproductive tract, 311 of whom were younger than 40 years. The dose given to the ovaries was estimated at $0.8 \mathrm{~Gy}$ for women below the age of 20 years and 1.2 Gy for women aged 20-40. The total dose to the corpus uteri was 24 Gy and was an important factor affecting miscarriage incidence. After exposure to a dose between 4 and 7 Gy in 1-4 fractions using a 200-250 kV radiation source, permanent menopause was observed in 40-year-old women. The risk of permanent sterilization in 20-year-old women who were given a 20 Gy dose in the period of 6 weeks was assessed at 50\%. The risk decreased with the growing number of dose fractions [16]. 
Larsen et al. evaluated 100 women who underwent chemo- and/or radiotherapy during childhood. Mean age was 5.4 at diagnosis and 25.7 at entering the study. Fifty-six patients received radiotherapy. Of these, 49 patients received radiotherapy to one field, six patients to two fields, and one patient to three fields. Sixteen received cranial irradiation (median total dose, $24 \mathrm{~Gy}$ ), and 12 received irradiation to other fields above the diaphragm (median total dose, 37 Gy). Ten patients received total body irradiation (median total dose, $11.3 \mathrm{~Gy}$ ). Six patients received direct irradiation to the ovaries during the whole abdominal or pelvic irradiation (median total dose, $30.6 \mathrm{~Gy}$ ). Twenty patients received radiotherapy by other fields below the diaphragm (median total dose, $30.1 \mathrm{~Gy}$ ), and the ovaries could potentially have received indirect irradiation/scatter. Seventeen of the patients required hormonal substitution. The loss of follicular cells or ovarian atrophy was found, and follicle-stimulating hormone (FSH) and LH levels were below detectable values. Seventy women with spontaneous menstrual cycles had a smaller ovarian volume than the control group $\left(4.8 \mathrm{~cm}^{3}\right.$ vs. $\left.6.8 \mathrm{~cm}^{3}, p<0.001\right)$ and a lower number of cells per ovary (7.5 vs. $11, p<0.001)$. Additionally, the number of cells was inversely correlated with the dose received per ovary, use of alkylating neoplastic agents and older age at diagnosis [7, 17].

In their study, Doll and Smith found that $98 \%$ of 2069 patients receiving an ovarian dose of 3.6-7.2 Gy in 2-4 fractions had menstruation disorders. It is known that in the treatment of Hodgkin's disease, where areas of radiation included ovaries, administering a dose of 35 Gy leads to irreversible changes in ovarian cells. Ovarian transposition before starting radiation and ovarian shielding during radiotherapy influenced fertility and reduced the received dose to 6 Gy in 12-45 days, which improved the proportion of menstruation disorders to less than $50 \%[4,18]$.

In a multicenter study, Chemaitilly et al. analyzed 3390 women in terms of acute ovarian failure (AOF) incidence and in order to identify risk factors. They found that older age at diagnosis and radiation to abdomen and/or pelvis were associated with AOF (OR 1.8, $p<0.0001)$ and (OR 25.4, $p<0.0001$ ). Among the patients with AOF, 116 women (54\%) received a dose of 10 Gy to the ovarian area, which suggests that AOF develops in a small group of patients, especially among those who received a high radiation dose to the ovaries [7, 19].

\section{Corpus uteri}

Women who have undergone radiotherapy of the abdomen and/or pelvis are at greater risk of pregnancy complications, including problems with conceiving, spontaneous miscarriages, premature birth, low birth weight, placental disorders or perinatal newborn death [7]. In premature girls the uterus is more sensitive to radiation injury than in the adults. Doses leading to uterus dysfunction are estimated at 14-30 Gy [7, 12, 20, 21].

Patients with retained ovarian function, who had the uterus exposed to radiation, have postradiation changes that significantly affect conceiving and/or carrying a pregnancy to full term. These changes depend on radiation dose, tissue volume exposed to radiation, patient's age and time after treatment [4].

Critchley et al. compared patients exposed to radiation in childhood with women with no history of radiation exposure. It was found that uteri of the women who underwent radiotherapy were smaller and had fibrotic lesions. Additionally, there were changes in vascularization, and the endometrium did not change in response to estradiol and progesterone. Doppler ultrasonography examination showed little or no arterial vascularization of these uteri. The cause of these disorders is not clear: we do not know whether they are associated with microvascular injury or hormonal impairment. The author suggests that women who received radiation in childhood are not able to conceive and maintain pregnancy, even with the help of assisted reproductive technology [22].

In another study, by Larsen et al., the effect of radiotherapy on uterine volume was evaluated using transvaginal ultrasonography in 100 patients treated in childhood. The patients were divided into groups depending on the irradiated area: control group - no previous radiotherapy (44 women, uterine volume was $47 \mathrm{ml}$ ), history of radiotherapy to area above diaphragm (21 women, uterine volume was $40 \mathrm{ml}$ ), with history of radiotherapy to area below diaphragm (19 women, uterine volume $34 \mathrm{ml}$ ), and history of radiotherapy to uterus (16 women, uterine volume $13 \mathrm{ml}$ ). In 13 nulliparae with a history of radiotherapy directly to the uterus, smaller uterus was statistically correlated with younger age at radiation exposure $(p=0.02)$. Additionally, there was a statistically higher number of miscarriages during the $2^{\text {nd }}$ trimester in patients with history of radiotherapy to the uterus in comparison to women with no history of radiation exposure $(p=0.07)[7,23]$.

The case reports indicate that in women who underwent total body irradiation (TBI), uterine muscle thickness was considerably lower, with a tendency to uterine ruptures [7, 21].

Holm et al. evaluated using duplex ultrasound examination the effect of TBI radiotherapy on uterine volume and vascularization. The study included 12 patients treated for leukemia during childhood. Median age was 12.7 months in the period of 4 to 10.9 years after the TBI procedure. Median observation time was 21.5 years. The authors reported that median deviation of uterine volume was 2.6 when compared to the control group (range -6.3 to -0.6, $p=0.002$ ). Uterine blood flow decreased, with systolic blood flow in 6 of 9 patients, and diastolic blood flow was visible only in 1 of 9 patients. In contradistinction to this, in the control group diastolic blood flow was present in 35\% of women before puberty and in 100\% of adults [7, 24].

Green et al. assessed fetal loss in 1915 women who in the years 1970-86 were treated for neoplastic disease. As many as 4029 pregnancies were reported, 2349 (58\%) of them after completing radiotherapy. Relative risk (RR) of miscarriage was 1.40 for women who underwent radiotherapy to the skull in comparison to women with no history of radiotherapy ( $95 \% \mathrm{Cl}: 1.02-1.94)$. In women who underwent radiotherapy to the skull and spinal cord area, the relative risk $\mathrm{RR}$ of miscarriage was 2.22 (95\% Cl: 1.7-7.78) in comparison to women with no history of radiotherapy. 
Apart from that, the authors observed a tendency to increase in the risk of miscarriage in women whose ovaries were in or near the radiation field ( $R R 1.86, p=0.14$ ), up to $5 \mathrm{~cm}$ from the margin of the radiation field (RR 1.64, $p=0.06)$ in comparison to patients with no history of radiotherapy. The risk of miscarriage was not increased when the ovaries were shielded (RR 0.90, $p=0.86$ ). Additionally, a higher risk of low birth weight was observed in newborns of patients who underwent radiotherapy of the lesser pelvis (RR 1.85, $p=0.03$ ) [7, 25].

Signorello et al. conducted a similar study, but focused on potential increase in the risk of premature birth and/or intrauterine growth restriction. In this study, 1264 women who underwent treatment in the years 1968-2002 were analyzed. A relationship between high radiotherapy dose ( $>5 \mathrm{~Gy}$ ) to the uterine area and increase in the incidence of premature births $(\mathrm{OR}=3.5,95 \% \mathrm{Cl}$ : $1.5-8.0)$, low birth weight $(\mathrm{OR}=6.8,95 \% \mathrm{Cl}: 2.1-22.2)$ and low gestational age $(\mathrm{OR}=4.0,95 \% \mathrm{Cl}: 1.6-9.8)$ was found, when compared to women with no history of radiotherapy. Additionally, the risk increased with dose to the lower part of the uterus and was 50 cGy for premature birth and 250 cGy for low birth weight [26].

\section{Prevention}

Fertility preservation options vary by age and sex. For prepubescent males some centers offer cryopreservation of testicular tissue, but this method is still in the experimental stages. Potential future options include in vitro maturation of spermatogonia into spermatocytes or germ-cell transplant into native testicular tissue [27, 28].

For postpubertal males an effective method of fertility preservation is sperm cryopreservation. The semen sample can be obtained mainly through masturbation or testicular biopsy. Three collections with an interval of 48 hours in between collections to allow sperm to reaccumulate are recommended. Specimens can be stored for years and then can be used for intrauterine insemination or in vitro fertilization [27, 28].

In females an option for fertility conservation is storage by cryopreservation of embryos, oocytes or ovarian tissue. Postpubertal women can undergo gonadotropin stimulation of the ovaries, followed by oocyte or embryo cryopreservation. During stimulation follicle development is observed via estradiol levels and ultrasound measurements of follicle size. Mature oocytes are removed from the ovaries by transvaginal ultrasound-guided aspiration supported by light anesthesia. Cancer treatment can often be initiated the next day. Oocytes can be combined with sperm to create embryos or cryopreserved in an unfertilized state. Embryo cryopreservation is the most mature technology available and is the most effective strategy to date [29].

Ovarian tissue cryopreservation is currently available but is considered experimental, because of difficulties in recovering and using immature oocytes. This technique can be used for adults and for children. Laparoscopy is required to undertake a biopsy of an ovary or to remove the whole ovary for preservation [30]. Tissue is cut into thin sections and then cryopreserved by slow freezing or vitrification. Potential future uses of the tissue include autotransplantation into the pelvis or a heterotopic site with natural ovulation or harvesting of oocytes for in vitro fertilization after gonadotropin stimulation [1]. Return of native tissue to the patient inspires fears of the possibility of reintroduction of malignant cells [31].

Ovarian transposition is another possibility to conserve fertility in females. It is a procedure performed in order to transpose an ovary out of the radiation field for the duration of therapy. It was introduced in the 1950s and is applied in the settings of cervical cancer, rectal cancer, Hodgkin and non-Hodgkin lymphoma and childhood sarcoma radiotherapy. The procedure consists of relocating the ovary and part of the Fallopian tube superolaterally as far as possible, most often above the pelvis, at the L4-L5 level. In patients receiving radiation to the pelvic field and with Hodgkin's disease receiving inverted $Y$ radiation, the ovary is transposed medially [7, 32, 33].

Covens et al. assessed the radiation dose received by transposed ovaries in patients treated for cervical cancer. He estimated that the median dose was 1.26 Gy for intracavitary brachytherapy and 1.35-1.90 Gy for teleradiotherapy to the pelvis [7, 34].

Morice et al. evaluated the efficacy and complications of bilateral ovarian transposition in 104 patients. A total of 59 patients had intracavitary mono-brachytherapy up to a dose of $60 \mathrm{~Gy}$, while 25 had pelvic teleradiotherapy of a total dose of 45 Gy combined with chemotherapy (cisplatin) and a brachytherapy boost (increase in the dose) of 15 Gy. Ovarian function was assessed using ultrasonography and sex hormone determination. Median observation time was 31 months. The authors concluded that organ function preservation occurred in $90 \%$ of women who had only brachytherapy and $60 \%$ who had combined treatment. Complications associated with transposition included: ovarian cysts $-23 \%$, chronic pain $-3 \%$, ovarian metastases $-1 \%$. Other complications included: vascular injuries, fallopian tube rupture and migrating ovary [7, 34, 35].

Kuohung et al. analyzed 15 women who at age 5-14 had cerebrospinal axis radiation therapy with unilateral ovarian transposition due to medulloblastoma. A comparison with a control group who had radiotherapy without ovarian transposition showed a lower proportion of ovarian dysfunction, such as increased FSH level or permanent absence of menstruation (13\% vs. $45 \%, p=0.09)[7,36]$.

\section{Summary}

The optimal approach to fertility preservation in patients with neoplastic disease depends on age, patient's will and time from antineoplastic therapy. All the possibilities of fertility preservation should be considered, such as ovarian transposition, gonadal shielding, oocytes and ovarian tissue saving through cryopreservation, embryo cryopreservation (depending on national regulations) or ovarian autotransplantation to upper limb with creation of vascular anastomoses. It should be emphasized that the physician's duties before starting antineoplastic ther- 
apy include starting a discussion concerning fertility preservation, procreative capacity and having children, even if the patient does not think about it in the face of illness, or is a child who is unaware of neoplastic disease therapy repercussions [7].

Infertility as a result of antineoplastic therapy is becoming a very important issue due to the growing incidence of neoplastic diseases. Routinely applied antineoplastic therapy methods and the illness itself lead to fertility disorders [5]. Fertility preservation procedures usually require at least 2-3 weeks, so immediate referral of the patient to an appropriate specialist and centre is crucial [1].

The time to start efforts to get pregnant after completing the treatment is unknown. Many experts recommend a 2-year period due to the highest risk of disease recurrence at this time. Women receiving complementary hormone therapy should extend this time period to 5 years, which is often hard to accept for patients and their partners [1].

The progress in antineoplastic therapy improves treatment results, but at the same time requires a deeper look at existential needs of the patient. Reproductive function is an integral element of self-esteem and should be taken into account during therapy planning [38].

\section{The authors declare no conflict of interest.}

\section{References}

1. Jensen J, Morbeck D, Coddingron C. Fertility preservation. Mayo Clin Proc 2011; 86: 45-9.

2. Schover L, Rybicki L, Martin B, Bringelsen KA. Having children after cancer. A pilot survey of survivors' attitudes and experiences. Cancer 1999; 86: 697-709.

3. Meirow D. Ovarian injury and modern options to preserve fertility in female cancer patients treated with high dose radio-chemotherapy for hemato-oncological neoplasias and other cancers. Leuk Lymphoma 1999; 33: 65-76.

4. Ogilvy-Stuart A., Shalet S. Effect of radiation on the human reproductive system. Environ Health Perspect 1993; 101: 109-16.

5. Mazur-Roszak M, Tomczak P, Litwiniuk M. Oncology and infertility: selected issues. Part I. Przyczyny zaburzeń płodności. Contemp Oncol (Pozn) 2005; 9: 26-29.

6. Rowley M, Leach D, Warner G, et al. Effect of graded doses of ion izing radiation on the human testis. Radiat Res 1974; 59: 665-78.

7. Wo J, Viswanathan A. Impact of radiotherapy on fertility, pregnancy, and neonatal outcomes in female cancer patients. Int J Radiat Oncol Biol Phys 2009; 73: 1304-12.

8. Hahn E, Feingold S, Nisce L. Aspermia and recovery of spermatogenesis in cancer patients following incidental gonadal irradiation during treatment: a progress report. Radiology 1976; 119: 223-5.

9. Speiser B., Rubin P., Casarett G. Aspermia following lower truncal irradiation in Hodgkin's disease. Cancer 1973; 32: 692-8.

10. Tsatsoulis A, Shalet S, Morris I, de Kretser DM. Immunoactive inhibin as a marker of Sertoli cell function following cytotoxic damage to the human testis. Horm Res 1990; 34: 254-9.

11. Faddy M, Gosden R, Gougeon A, Richardson SJ, Nelson JF. Accelerated disappearance of ovarian follicles in mid-life: implications for forecasting menopause. Hum Reprod 1992; 7: 1342-6.

12. Bath L., Critchley H., Chambers S, Anderson RA, Kelnar CJ, Wallace WH. Ovarian and uterine characteristics after total body irradiation in childhood and adolescence: response to sex steroid replacement. Br J Obstet Gynaecol 1999; 106: 1265-72.
13. Wallace W, Shalet S., Hendry J, Morris-Jones PH, Gattamaneni HR. Ovarian failure following abdominal irradiation in childhood: the radiosensitivity of the human oocyte. Br J Radiol 1989; 62: 995-8.

14. Wallace $W$, Thomson A, Kelsey $T$. The radiosensitivity of the human oocyte. Hum Reprod 2003; 18: 117-21.

15. 15. Wallace W, Thomson A., Saran F, Kelsey TW. Predicting age of ovarian failure after radiation to a field that includes the ovaries. Int J Radiat Oncol Biol Phys 2005; 62: 738-44.

16. Dickson $\mathrm{R}$. The late results of radium treatment for benign uterine haemorrhage. Br J Radiol 1969; 42: 582-94.

17. Larsen E, Muller J, Schmiegelow K, Rechnitzer C, Andersen AN. Reduced ovarian function in long-term survivors of radiation-and chemotherapy-treated childhood cancer. J Clin Endocrinol Metab 2003; 88: 5307-14

18. Doll R, Smith $\mathrm{P}$. The long-term effects of $\mathrm{x}$ irradiation in patients treated for metropathia haemorrhagica. Br J Radiol 1968; 41: 362-8.

19. Chemaitilly W, Martens A, Mitby P, et al. Acute ovarian failure in the childhood cancer survivor study. J Clin Endocrinol Metab 2006; 91: 1723-8.

20. Green D, Peabody E, Nan B, Peterson S, Kalapurakal JA, Breslow NE. Pregnancy outcome after treatment for Wilms tumor: a report from the National Wilms Tumor Study Group. J Clin Oncol 2002; 20: 2506-13.

21. Norwitz E, Stern H., Grier H, et al. Placenta percreta and uterine rupture associated with prior whole body radiation therapy. $\mathrm{Ob}$ stet Gynecol 2001; 98: 929-31.

22. Critchley $\mathrm{H}$, Wallace $\mathrm{W}$, Shalet S. et al. Abdominal irradiation in childhood; the potential for pregnancy. Br J Obstet Gynaecol 1992; 99: 392-394.

23. Larsen E, Schmiegelow K, Rechnitzer C, et al. Radiotherapy at a young age reduces uterine volume of childhood cancer survivors. Acta Obstet Gynecol Scand 2004; 83: 96-102.

24. Holm K, Nysom K, Brocks V, et al. Ultrasound B-mode changes in the uterus and ovaries and Doppler changes in the uterus after total body irradiation and allogeneic bone marrow transplantation in childhood. Bone Marrow Transplant 1999; 23: 259-63.

25. Green DM, Whitton J, Stovall M, et al. Pregnancy outcome of female survivors of childhood cancer: a report from the Childhood Cancer Survivor Study. Am J Obstet Gynecol 2002; 187: 1070-80.

26. Signorello L, Cohen S, Bosetti C, et al. Female survivors of childhood cancer: preterm birth and low birth weight among their children. J Natl Cancer Inst.2006; 98: 1453-61.

27. Bączkowski T, Kurzawa R. Profilaktyka niepłodności w chorobach onkologicznych. Przewodnik Lekarza 2001; 11: 195-200.

28. Ajala T, Rafi J, Larsen-Disney P, Howell R. Fertility preservation for cancer patients: a review. Obstet Gynecol Int 2010; 2010: 160386.

29. Oktay K, Cil AP, Bang H. Efficiency of oocyte cryopreservation: a meta-analysis. Fertil Steril 2006; 86: 70-80.

30. Oktay K, Buyuk E. Ovarian transplantation in humans: indications, techniques and the risk of reseeding cancer. Eur J Obstet Gynecol Reprod Biol 2004; 113 Suppl 1: S45-7..

31. Abir R, Nitke S, Ben-Haroush A, Fisch B. In vitro maturation of human primordial ovarian follicles: clinical significance, progress in mammals, andmethods for growth evaluation. Histol Histopathol 2006; 21: 887-98.

32. Mc C., Keaty E., Thompson J. Conservation of ovarian tissue in the treatment of carcinoma of the cervix with radical surgery. Am J Obstet Gynecol 1958; 75: 590-600.

33. Sella T, Mironov S, Hricak H. Imaging of transposed ovaries in patients with cervical carcinoma. AJR Am J Roentgenol 2005; 184: 1602-10.

34. Covens AL, van der Putten HW, Fyles AW, Leung PM, O'Brien PF, Murphy KJ, DePetrillo AD. Laparoscopic ovarian transposition. Eur Jynaecol Oncol 1996; 17: 177-82.

35. Morice P, Juncker L, Rey A, El-Hassan J, Haie-Meder C, Castaigne D. Ovarian transposition for patients with cervical carcinoma treated by radiosurgical combination. Fertil Steril 2000; 74: 743-8.

36. Kuohung W, Ram K, Cheng DM, Marcus KJ, Diller LR, Laufer MR. Laparoscopic oophoropexy prior to radiation for pediatric brain tumor and subsequent ovarian function." Hum Reprod 2008; 23: 117-21. 
37. Kroman N, Jensen MB, Wohlfahrt J, Ejlertsen B; Danish Breast Can cer Cooperative Group. Pregnancy after treatment of breast cancer - a population-based study on behalf of Danish Breast Cancer Cooperative Group. Acta Oncol 2008; 47: 545-9.

38. Mazur-Roszak M, Tomczak P, Litwiniuk M, Markowska J. Oncology and infertility: selected issues. Part II. Preservation of the reproductive function. Contemp Oncol (Pozn) 2005; 9: 65-8.

\section{Address for correspondence}

Marta Biedka MD PhD

Radiotherapy Department 1

Oncology Centre in Bydgoszcz

Romanowskiej 2

85-796 Bydgoszcz, Poland

tel. +48523743374

e-mail: martabiedka@tlen.pl

Submitted: 14.02 .2014

Accepted: 12.01 .2015 